\title{
Size constancy in extended haptic space
}

\author{
CHARLES HANLEY \\ Michigan State University, East Lansing, Michigan 48823 \\ and \\ DAVID P. GOFF \\ University of Iowa, Iowa City, lowa 52240
}

\begin{abstract}
When a stick is used to extend the boundaries of the world perceived by touch, haptic parallels to visual angle and size constancy appear. In a pilot study, Ss using a stick to feel an out-of-reach gap were more likely to underestimate its size than were Ss feeling a nearby gap with the index finger, but size constancy was relatively high with the stick. The main study, comparing judgments of gaps at intermediate and far positions, confirmed these findings. Errors were greater for the more distant gap, but constancy was high in both positions. Correlations between far and intermediate judgments indicated that $\mathrm{Ss}$ were consistent in the type of error made. Similar findings emerged from a partial replication with blind and partially sighted Ss.
\end{abstract}

Haptic space, the world we perceive by touch, normally ends at our extremities. But when we use a stick or cane as a probe, we extend haptic space to include things that are ordinarily out of reach. Subjective experience in such cases, Gibson (1966) notes and Vaught, Simpson, and Ryder (1968) confirm, locates the feeling of contact in the tool rather than in the hand holding it. An objective side of this experience, the accuracy of perception of size when touch is mediated by a stick, is the topic of the present investigation.

While haptic space has many features in common with visual space, achievement of size constancy is simpler for normal haptic space. From physical contact with an object, stimulus input is the same as far as the fingers or hands alone are concerned whether the object being touched is next to the body or at arm's length. Stimulus input to the eye, on the other hand, varies with the distance of the object looked at. Under most conditions, then, there is no haptic equivalent of the change in visual angle that accompanies a change in the distance between object and 0 . Use of a tool to extend the range of touch, however, introduces this angular relation into haptic space. To feel out size with a stick, we act as though the stick were a conveniently elongated finger, waggling it the way we might move a real finger to determine the bore of a knothole. In this sort of employment, the finger swings through an angle whose apex is in the knuckle at the base of the finger, and whose sides are tangential to the circumference of the hole. The stick sweeps out a similar angle, except that its apex may be in the palm, wrist, elbow, or even in the shoulder. By its swings, the stick marks out a "haptic angle," and this angle parallels visual angle exactly with respect to distance: the farther an object, the smaller its haptic and visual angles. Achievement of size constancy in extended haptic space thus must require some kind of allowance for the decrease in haptic angle that accompanies increased distance of the object being touched, an allowance parallel to that required in vision.

\section{METHOD}

A pilot study, a main study, a partial replication with blind Ss, and a control study dealt with perceived widths of gaps felt with the index finger or with a stick.

\section{Subjects}

Ss in the pilot study were 27 male and 25 female undergraduates from introductory psychology classes at Michigan State University, which also provided the 22 males and 42 females in the main study. In the replication, the 9 male and 6 female college students were all legally blind, 8 totally and 7 partially. The control study employed 19 males and 23 females from the introductory classes.

\section{Procedure}

$S$ sat on an adjustable chair at the end of a wooden table, its top at elbow height. Figure 1 shows the dimensions of the stick, a 41-g wooden dowel, plus the position of the standard gap and the three possible locations of the variable gap for right-handed Ss. For left-handers, arrangements were mirror-image reversals. The gaps were within pairs of metal rods, each rod $10 \mathrm{~cm}$ long and $5 \mathrm{~mm}$ thick.

Wearing opaque goggles, $S$ sat with hands on low blocks mounted at the end of the table to bring the base of the hands level with the base of the gaps. The blocks made it easy for $E$ to check that the hands were in the same position on each trial. S felt the standard gap with the index finger so placed that contact with the outer of the pair of rods came at the base of the fingernail. The preferred hand was in a comparable position facing the variable gap, which was felt either with the index finger or with the wooden stick that $S$ had seen and handled previously. Under these arrangements, finger or stick swung inside the gap with the side of the stationary hand as a pivot. To determine whether restriction of hand movement might have reduced perceptual accuracy, Ss in the control study felt the gaps without instructions as to hand placement.

The standard gap was either 2.5 or $4.5 \mathrm{~cm}$ and was always in the near position. The variable gap changed by $5-\mathrm{mm}$ steps, 


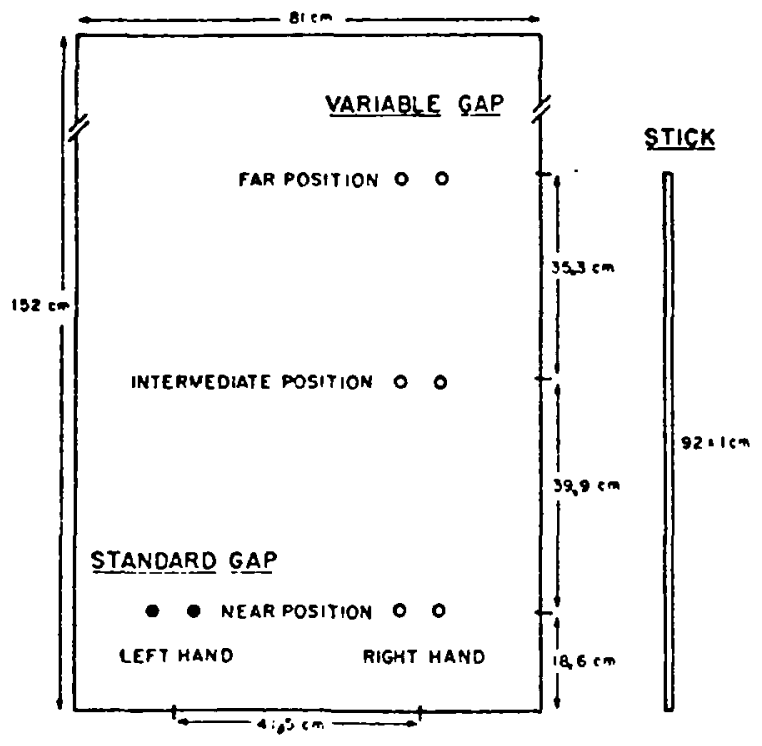

Fig. 1. Standard and variable stimuli arranged on table for right-handed $S$.

except in the pilot study, where 2-mm steps were employed when both fingers were used.

The pilot study compared finger-finger and finger-stick judgments with the variable stimulus in the far position for the latter condition; Ss were randomly assigned to one of four groups in a factorial design involving the two standards and the two manipulative conditions. Ss in the main study made finger-stick judgments of one of the two standards, the variable first at the far and then at the intermediate position. Blind Ss followed this procedure, but only with the $4.5-\mathrm{cm}$ standard. For the control study, Ss, free to vary hand position, first employed the stick to judge the variable gap at the far position, then stretched to use the index finger of the preferred hand to judge the variable at the intermediate position; half judged the smaller, half the larger standard. In both parts of the control study, the standard gap was felt with the nonpreferred index finger in whatever manner $S$ chose. Finally, in all judgments using the stick in these studies, $S$ rested it on the table when $E$ adjusted the variable gap for each new trial.

\section{Size Constancy}

For judgments with the stick, size constancy was obtained from a Brunswik ratio based on mean point of subjective equality (PSE) from five ascending and five descending two-category, method-of-limits series, these preceded by single ascending. and descending practice series. A value of 1.00 for the ratio shows perfect constancy, a value of 0.00 represents zero constancy. The ratio for size constancy (Brunswik, 1956) is given by $(P-r) /(P-B)$, where $r$ is the magnitude of the empirical PSE, $B$ the size of the standard stimulus, and $P$ the magnitude of the PSE at zero constancy, where a hypothetical $S$ judges the distant and nearby gaps equal when their haptic angles are equal, just as a similar $S$ trying to match the physical size of near and far objects in a vision study would match their retinal images instead. Figure 2 presents these relations schematically. Note that the Brunswik ratio measures relative constant error, not random error or difference limen.

The value of $P$ in the present investigation was obtained empirically. For the pilot study, E moved the standard gap to the same side as the variable (to $C$ in Fig. 2), replaced the variable gap with a metric ruler, and, acting as his own $S$, swung the stick from one side of the standard gap to the other, noting the excursion of the stick on the ruler to measure $P$, the chord of the haptic angle at that location. By a similar procedure in the main study and replication, two chords of the angle were taken for every $S$, one at the far and one at the intermediate position, each after completion of the corresponding set of width judgments. Each chord was the average of three measurements.

\section{RESULTS}

In the pilot study, the finger-finger conditions established a base line for absolute accuracy of perception of width in ordinary haptic space: mean PSE was $2.45 \mathrm{~cm}$ for the $2.5-\mathrm{cm}$ standard and $4.33 \mathrm{~cm}$ for the $4.5-\mathrm{cm}$ standard gap, neither PSE differing significantly from its standard. For finger-stick conditions-near standard and far variable-mean PSE was $2.90 \mathrm{~cm}$ for the smaller standard and 5.57 for the larger, each PSE differing from its standard at the .05 level. The corresponding Brunswik ratios were 0.95 and 0.94. Perception was less accurate in extended haptic space, but the increase in error through use of the stick was not large relative to the possibilities of error.

The main study and replication confirmed the findings of the pilot study. Table 1 shows PSE, chord, and Brunswik ratio data for normal and blind Ss at intermediate and far positions and with each of the two standards. The data from normals are consistent with those from the pilot study, and results for blind Ss are similar to those from Ss with normal vision. Medians rather than means appéar in Table 1 , because four normal and one blind' $S$ had incomplete series of judgments of the $4.5-\mathrm{cm}$ standard, reaching the limits of

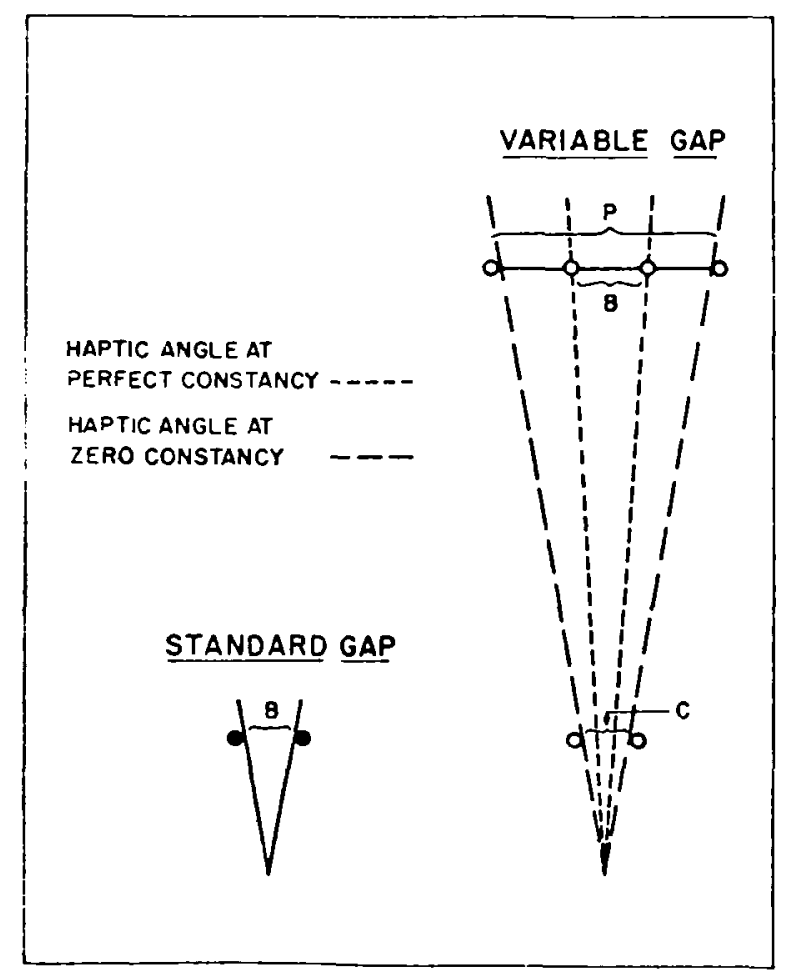

Fig. 2. Haptic angle and size constancy. 
Table 1

PSE, Chord, and Brunswik Ratio for Width Judgments by Normal and Blind Subjects: Median and Interquartile Range

\begin{tabular}{|c|c|c|c|c|c|c|c|}
\hline \multirow{3}{*}{$\begin{array}{l}\text { Standard } \\
(\mathrm{cm})\end{array}$} & \multirow[b]{3}{*}{ Ss } & \multicolumn{6}{|c|}{ Position of Variable } \\
\hline & & \multicolumn{3}{|c|}{ Intermediate } & \multicolumn{3}{|c|}{ Far } \\
\hline & & PSE & Chord & BR & PSE & Chord & BR \\
\hline & & \multicolumn{6}{|c|}{ Median } \\
\hline 2.5 & 32 Normal & 2.55 & 7.9 & 0.99 & $3.10^{*}$ & 13.3 & 0.96 \\
\hline 4.5 & 32 Normal & 4.72 & 15.3 & 0.98 & $5.52^{*}$ & 26.4 & 0.95 \\
\hline 4.5 & 15 Blind & 4.60 & 15.3 & 0.97 & 5.10 & 23.0 & 0.95 \\
\hline & & \multicolumn{6}{|c|}{ Interquartile Range } \\
\hline 2.5 & 32 Normal & 0.95 & 3.14 & 0.14 & 0.82 & 4.80 & 0.08 \\
\hline 4.5 & 32 Normal & 1.50 & 5.40 & 0.10 & 1.53 & 5.55 & 0.06 \\
\hline 4.5 & 15 Blind & 2.03 & 3.50 & 0.09 & 1.55 & 4.80 & 0.12 \\
\hline
\end{tabular}

${ }^{*}$ Different from standard at .02 level

the apparatus, which could not present gaps greater than $9 \mathrm{~cm}$. In these five cases of low constancy, PSEs were not computed from the incomplete data. Three of these Ss were off-scale only at the far position and had Brunswik ratios of $0.71,0.74$, and 0.78 in the near position. As might be expected when average Brunswik ratios approach unity, there were cases of over-constancy, the highest being 1.29, based on a PSE of $1.75 \mathrm{~cm}$ for the $2.5-\mathrm{cm}$ standard at the near position.

Evidence of the reliability of the measures of PSE, chord, and Brunswik ratio appears in Table 2 as product-moment correlations between the intermediate and far positions. ${ }^{1}$ Degree of deprivation of vision among the blind Ss was not associated with differences in the performance-data for the blind and partly sighted were quite similar on all measures shown in these tables.

Under conditions of the present investigation, size constancy was high but not perfect, the average $S$ tending to match a nearby gap with a slightly larger distant one. Data from the control study related to the possibility that constancy may be lower at the limits of normal haptic space, so that the error does not involve haptic angle at all, and to the chance that fixing hand position-necessary for obtaining Brunswik ratios-reduces constancy via elimination of information that stems from movement. When Ss stretched to feel the intermediate gap with the finger, median PSEs for the 2.5 - and $4.5-\mathrm{cm}$ standards were 2.85 and $4.50 \mathrm{~cm}$, respectively, offering little evidence of any general tendency to overestimate the size of a gap at the limit of ordinary haptic space. When Ss used the stick freely to feel the far gap, median PSEs for the smaller and larger standards were 3.40 and $5.60 \mathrm{~cm}$, respectively, both

Table 2

Correlations Between Measures from Intermediate and Far Positions of Standard

\begin{tabular}{ccccc}
$\begin{array}{c}\text { Standard } \\
(\mathrm{cm})\end{array}$ & Ss & PSE & Chord & $\begin{array}{c}\text { Brunswik } \\
\text { Ratio }\end{array}$ \\
\hline 2.5 & 32 Normal & .72 & .79 & .65 \\
4.5 & 28 Normal & .68 & .75 & .65 \\
4.5 & 14 Blind & .77 & .52 & .82 \\
\hline
\end{tabular}

slightly larger than corresponding values in Table 1 . All Ss switched the stick from relatively fixed hand positions, but tended to keep the preferred hand slightly forward of its mate, some lifting the hands from the table while making judgments. The few who tried keeping both hands rigid in order to move finger and stick in parallel quickly abandoned that strategy.

\section{DISCUSSION}

There are two ways to evaluate the accuracy of the use of the stick in this study: to consider oniy the magnitude of the error, or to look at error in relation to what it might have been in the absence of perceptual constancy-the approach expressed by the Brunswik ratio. In terms of magnitude of error, use of the stick, when the variable was in the intermediate position, led to a small average overestimation of the standard of about the same extent as the average underestimation of the standard in the pilot study, where the finger was used to feel the variable in the near position. At the far position, however, the stick led to an overestimation of the standard by approximately one-fourth. This increase in the size of the error as the distance between standard and variable increased could occur because of an angular error, and, in support of this view, the correlations in Table 2 show that Ss are consistent in the direction and relative magnitude of their errors. If the average $\mathrm{S}$ consistently overestimates the haptic angle needed for an accurate judgment, the farther away the variable gap, the more PSE will exceed the standard.

Yet, in relative terms, results for the two positions of the variable stimulus are not very different. Both Brunswik ratios are high; this is because the zero-constancy value of PSE-the chord-also increases with an increase in the distance between standard and variable, so that a small error for nearby objects has the same effect on the Brunswik ratio as a large error for far objects.

It would be a mistake, nevertheless, to assume that error must originate in the estimation of the haptic angle. Underestimation of the distance of the variable 
has a parallel effect. The nearer the gap to $S$, the wider the angle a stick must swing through to touch both sides of the gap. If distance is underestimated, $S$ will sweep too great an angle and set PSE too large. Naturally, errors in estimation of both angle and distance can combine to cancel or reinforce each other. Ss in this investigation handled, and all but the blind saw, the stick before using it. In addition, they rested it on the table between trials, getting information about its characteristics from picking it up and putting it down and inadvertently touching parts of the apparatus. Under the freer conditions of the control study, no S diverged much from the stricter conditions; variations that occurred appeared to be matters of comfort rather than information seeking. Thus, $\mathrm{S}$ might consistently keep the hand holding the stick somewhat forward of the other hand, or lift both hands from the table when judging.

The use of blind Ss in this study, of course, was a rough attempt to determine whether past experience affected accuracy of the judgments, but no differences related to blindness were apparent. In retrospect, it is easy to see that the task was as novel for the blind as for the sighted. Some of the blind Ss commented that their white canes served primarily as signals to others and shields for their own shins. Moreover, normal Ss may have had a certain amount of incidental learning in this area.
The $\mathrm{S}$ making these judgments has a second source of information besides the haptic system. Manipulations of the stick and, to a lesser extent, finger movements make sounds that might yield subtle cues to the sizes of the gaps. In real life, of course, the case is the same-a person both feels and hears the tools he uses. The sounds convey information already available from touch; whether this redundancy improves discrimination is an open question.

\section{REFERENCES}

Brunswik, E. Perception and the representative design of psychological experiments. Berkeley: University of California Press, 1956.

Gibson, J. J. The senses considered as perceptual systems. Boston: Houghton Mifflin, 1966.

Vaught, G. M., Simpson, W. E., \& Ryder, R. "Feeling" with a stick. Perceptual \& Motor Skills, 1968, 26, 848.

\section{NOTE}

1. Reliability of PSE is determined by the consistency of S's judgments, whereas reliability of the measurement of the chord is determined only by the consistency of S's movement of the stick and E's reading of the scale.

(Received for publication November 22, 1972; revision received August 29, 1973.) 